\title{
Blue-green to near-IR switching electroluminescence from Si-rich silicon oxide/nitride bilayer structures
}

\author{
Y. Berencén, ${ }^{1, *}$ O. Jambois, ${ }^{1}$ J. M. Ramírez, ${ }^{1}$ J. M. Rebled ${ }^{1,4}$ S. Estradée, ${ }^{1,5}$ \\ F. Peiró, ${ }^{1}$ C. Domínguez, ${ }^{2}$ J. A. Rodríguez, ${ }^{3}$ and B. Garrido ${ }^{1}$ \\ ${ }^{1}$ MIND-IN2UB, Dept. Electrònica, Universitat de Barcelona, Martí i Fanquès 1, 08028, Barcelona, Spain \\ ${ }^{2}$ Instituto de Microelectrónica de Barcelona (IMB-CNM, CSIC), Bellaterra 08193, Barcelona, Spain \\ ${ }^{3}$ Physics Faculty, University of Havana, San Lázaro y L, Vedado, 10400, Havana, Cuba \\ ${ }^{4}$ Institut de Ciència de Materials de Barcelona-CSIC, Campus UAB, Bellaterra 08193, Barcelona, Spain \\ ${ }^{5}$ TEM-MAT, CCiT-UB, Solé i Sabarís 1, 08028, Barcelona, Spain \\ *Corresponding author: yberencen@el.ub.es
}

Received March 2, 2011; revised May 27, 2011; accepted June 14, 2011; posted June 14, 2011 (Doc. ID 143511); published July 11, 2011

\begin{abstract}
Blue-green to near-IR switching electroluminescence (EL) has been achieved in a metal-oxide-semiconductor light emitting device, where the dielectric has been replaced by a Si-rich silicon oxide/nitride bilayer structure. To form $\mathrm{Si}$ nanostructures, the layers were implanted with Si ions at high energy, resulting in a Si excess of $19 \%$, and subsequently annealed at $1000^{\circ} \mathrm{C}$. Transmission electron microscopy and EL studies allowed ascribing the blue-green emission to the Si nitride related defects and the near-IR band with the emission of the Si-nanoclusters embedded into the $\mathrm{SiO}_{2}$ layer. Charge transport analysis is reported and allows for identifying the origin of this twowavelength switching effect. () 2011 Optical Society of America

OCIS codes: $250.0250,230.2090,230.6080,260.3800$.
\end{abstract}

Silicon-rich silicon oxide (SRSO) and silicon-rich silicon nitride (SRSN) have drawn great interest in the last decades as active dielectric matrices in optoelectronic devices [1-4]. Particularly, these materials have been used in the fabrication of low-cost light emitting devices compatible with the mainstream Si technology, They also provide a solution to the monolithic integration of electronic and optical technologies on the same Si chip. Different electroluminescence (EL) excitation mechanisms have been reported, such as hot electrons [5] and optically active defects [6] or field-effect EL using pulsed excitation [1]. The E $\bar{L}$ emission of the Si-nanocrystals (Si-ncs) embedded in $\mathrm{SiO}_{2}$ is usually in the red-IR and shows a narrow shift capability depending on Si-nc size $[1,3]$. Regarding Si nitrides, luminescence in the bluegreen is also reported, ascribed either to Si nitride related defects or Si-ncs [4,7]. In addition, some strategies have been employed in order to extend the EL emission to different spectral region. For instance, defects created by dopants such as rare-earth ions can lead to light emission from UV to near-IR, depending on their specific energy level structure [8].

In this Letter we report two-wavelength switching metal-nitride-oxide-semiconductor light emitting devices (MNOSLED) based on a SRSO/SRSN bilayer structure.

The devices are similar to metal-oxide-semiconductor field-effect transistors with a $100 \mathrm{~nm}$ thick polycrystalline silicon layer used as an optically transparent gate electrode. Since its transmittance spectrum peaks at $470 \mathrm{~nm}$ and $760 \mathrm{~nm}$ [3], the final EL spectra of the devices are modulated accordingly. In order to combine light emission from different matrices, a SRSO/SRSN bilayer structure was designed ad hoc to form the insulator of the transistor. The $\mathrm{Si}$ excess in the $\mathrm{SiO}_{2}$ and $\mathrm{Si}_{3} \mathrm{~N}_{4}$ layers was introduced by ion implantation. A subsequent annealing treatment at $1000{ }^{\circ} \mathrm{C}$ during one hour was performed to recover the matrices and precipitate the Si-ncs. The implantation parameters (energy and dose) were chosen by stopping and range of ions in matter simulations to obtain a Si excess of $19 \%$, attending to photoluminescence studies in previous works [7]. Energy filtered transmission electron microscopy (EFTEM) measurements were performed using a JEOL 2010-FEG $(200 \mathrm{kV})$ microscope equipped with a Gatan imaging filter for EFTEM imaging mode. Moreover, due to the importance of the proper localization of the geometry of the devices, the cross section was defined by a focused ion beam. (Dual Strata- FEI Company).

Electrical measurements using a semiconductor parameter analyzer (Agilent B1500A) and a probe station (Cascade Microtech Summit 11000) were accomplished at room temperature. Both source and drain were grounded during forward (or backward) bias, in order to improve the injection into the bilayer. By convention, it is considered that a negative direct current $(\mathrm{dc})$ voltage applied to the polysilicon electrode corresponds to forward polarization (accumulation regime). Room temperature EL spectra were measured through an Acton 2300i grating spectrometer and a cryogenically cooled PI Spec-10-100B/LN charge-coupled device. The final spectra were corrected for the spectral response of the optical system.

Figure 1(a) shows an EFTEM cross section picture of the bilayer structure of the device under study. The zone at the border of the polycrystalline silicon (pc-Si) is formed by a silicon nitride layer about $28 \mathrm{~nm}$ thick. No Si-ncs were observed inside this region, in accordance with Ref. [9]. Moreover, the zone between silicon nitride and crystalline silicon $(c-\mathrm{Si})$ is a silicon oxide layer about $13 \mathrm{~nm}$ thick. Si-ncs with a mean size of $3.8 \pm 0.5 \mathrm{~nm}$ and embedded into this material are clearly observed in the image. In Fig. 1(b) a typical scheme of the device structure is presented.

The $I$ - $V$ curves (Fig. 2) are quite symmetrical for both polarities in the whole range of dc voltages, which suggests that, (i) leakage current is mainly due to electrons, 


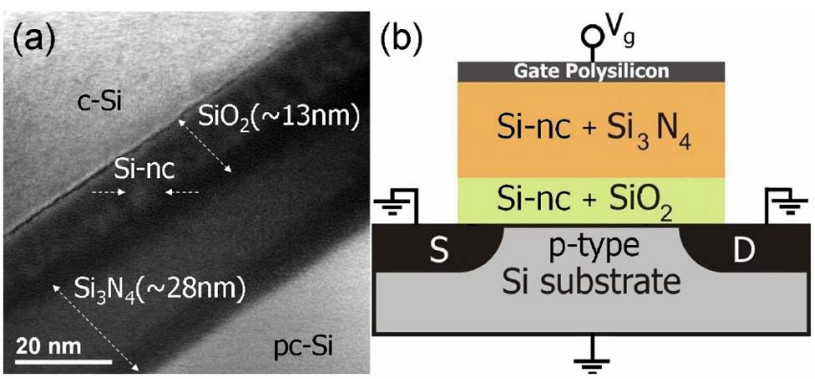

Fig. 1. (Color online) (a) Cross section EFTEM image showing the SRSO/SRSN bilayer and Si-ncs into the $\mathrm{SiO}_{2}$ layer. (b) Scheme of the light emitting device structure.

and (ii) the current is limited by the active layer and not by the electrode [10], although the dielectric structures are not symmetric. In addition, a strong saturation is observed at high voltages, attributed to the spatial charge formed at the interface between substrate or electrode (depending on the applied voltage) and the active layer, in agreement with previously published works [2]. The inset shows the good concordance of the experimental data, in accumulation and inversion regimes, with two different conduction mechanisms depending on the voltage values. At low voltages, the Poole-Frenkel (PF) mechanism is predominant, whereas at high voltages the space charge-limited current (SCLC) mechanism predominates. By fitting the experimental data to the theoretical expressions corresponding to these mechanisms [10] (see inset of Fig. 2), the average values obtained for the relative permittivity and the drift mobility in accumulation and inversion regime are $\varepsilon_{r}=7.2$ and $\mu=1.42 \times$ $10^{-5} \mathrm{~cm}^{2} / \mathrm{V} \cdot \mathrm{s}$. According to effective medium theory, the value of $\varepsilon_{r}$ can vary between 3.9 and 7.5, depending on the $\mathrm{SiO}_{2}$ and $\mathrm{Si}_{3} \mathrm{~N}_{4}$ permittivities, respectively. Therefore, this suggests that the $\varepsilon_{r}$-value deduced in the present work is physically correct. Likewise, the value of $\mu$ in our SRSO/SRSN bilayer is 1 order of magnitude lower than the value $\left(10^{-4} \mathrm{~cm}^{2} / \mathrm{V} \cdot \mathrm{s}\right)$ observed in the SRSN layer by Warga et al. [2], and 3 orders of magnitude higher than the value $\left(10^{-8} \mathrm{~cm}^{2} / \mathrm{V} \cdot \mathrm{s}\right)$ in the SRSO layer reported by Ryabchikov et al. [11]. This fact suggests the existence of a trade-off between the drift mobility values of the SRSN and SRSO layers, which indicates that the $\mu$-value obtained is physically acceptable. Consequently, the electron mobility into the bilayer used in the present work will be mostly limited by the SRSN layer.

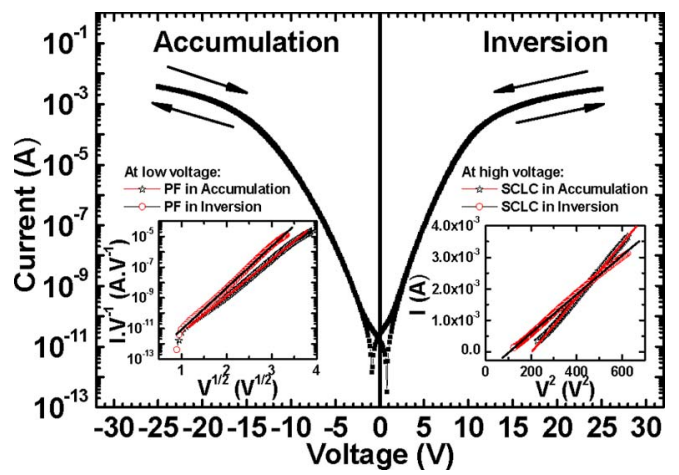

Fig. 2. (Color online) $I-V$ characteristic in accumulation and inversion. The inset shows the PF and SCLC fits in both regimes at low and high voltages, respectively.
An interesting switching effect is observed in Fig. 3, not reported before for MNOSLED based on SRSN/SRSO bilayer structure. By changing the dc voltage sign applied to the gate, the line-shape varies completely, thus leading to a switchable EL from a blue-green spectrum at positive dc voltage (inversion regime) to a near-IR spectrum at negative dc voltage (accumulation regime).

This difference can be understood by considering the radiative recombination processes taking place in each of the SRSN and SRSO single layers in both cases. At negative dc voltages, electrons are injected from gate to substrate through the bilayer following a PF conduction mechanism and, simultaneously, holes injection from the Si substrate into the few nanometer SRSO layer occurs [12]. (As mentioned above, no transport of holes is observed). Thus, the electrons find positively charged $\mathrm{Si}$ ncs embedded into $\mathrm{SiO}_{2}$, due to hole injection and the creation of excitons takes place (see left inset of the Fig. 4). Therefore, bipolar injection by direct tunneling in the SRSO leads to the near-IR light emission around $800 \mathrm{~nm}$ reported in the Fig. 3 , in accordance with previous results $[2,3]$, whereas the little blue-green peak $(\sim 535 \mathrm{~nm})$ in the same spectrum is ascribed to defects of the SRSN, such as Si-dangling bonds located in the middle of the $\mathrm{Si}_{3} \mathrm{~N}_{4}$ bandgap, as well as to bonding states of $\mathrm{Si}-\mathrm{Si}$ units that are close to the valence band edge $[4,13]$, which are optically activated by PF ionization. On the contrary, when a positive dc voltage is applied to the gate, the channel is formed in the $p$-type substrate when both source and drain are grounded, leading electrons to flow from the channel to the gate through Si-nc to Si-nc in the SRSO layer and defect to defect in the SRSN layer (see right inset of the Fig. 4). If voltage is large enough, the defects of the SRSN are optically activated, thus producing solely the blue-green light emission $(\sim 515 \mathrm{~nm})$, which is attributed to PF ionization. It implies that the excitation of the $\mathrm{Si}_{3} \mathrm{~N}_{4}$ defects, in this case, dominates over excitation of the Si-ncs into the $\mathrm{SiO}_{2}$ layer (emission at $\sim 800 \mathrm{~nm}$ ). This latter fact is only possible in an inversion regime due to the lack of injected holes from the electrode. Time-resolved EL measurements were

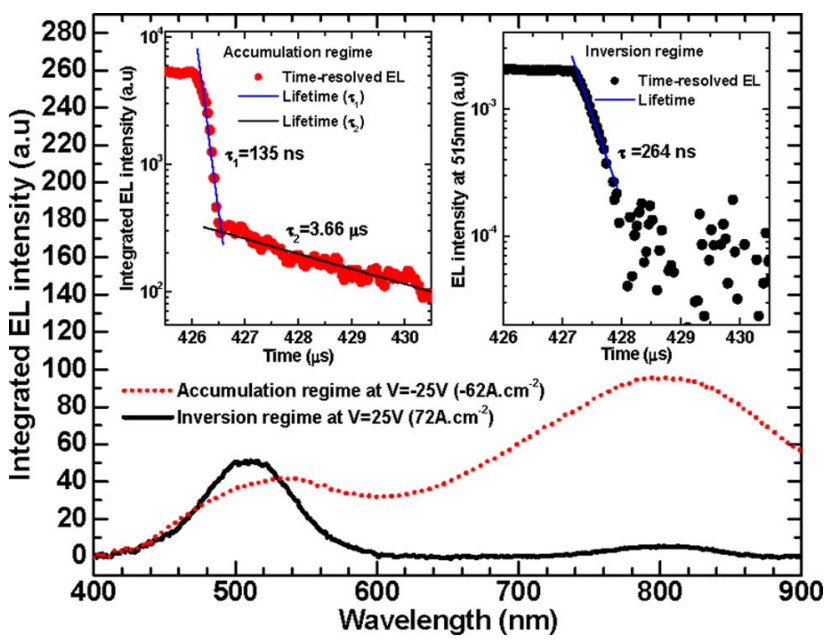

Fig. 3. (Color online) EL spectra for both gate polarities. Inversion regime (black solid line) and accumulation regime (red dot line). The inset shows the lifetimes of each luminescent species in both gate polarities. 


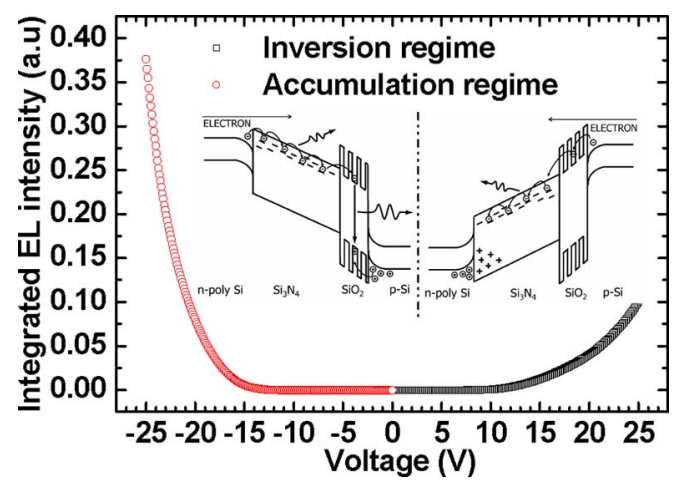

Fig. 4. (Color online) Integrated EL intensity versus voltage in accumulation and inversion regimes. The inset depicts the band diagram for both cases.

performed in both gate polarities using square pulses of $1 \mathrm{kHz}$. Two lifetimes (135 ns and $3.66 \mu \mathrm{s}$ ) were measured in accumulation (see left inset Fig. 3) ascribed to Si nitride related defects and excitonic recombination inside the Si-ncs, respectively. Whilst, in an inversion regime, solely a fastest lifetime was detected (see right inset Fig. 3), thus confirming the previous analysis. Moreover, in a typical Arrhenius plot (graph not shown) the activation energy of the traps (Si nitride related defects) drops as the driving voltage increases. This observation is consistent with a PF-type mechanism, where the thermal ionization is assisted by the applied electric field. The activation energy value found was $0.32 \mathrm{eV}$ at $5 \mathrm{MeV} / \mathrm{cm}$, which closely agrees with the value reported by Sze ( $0.64 \mathrm{eV}$ at the same electric field) [10] for a single silicon nitride films. Si-based light emitters using an $\mathrm{Eu}-$ implanted $\mathrm{SiO}_{2}$ single layer have been previously reported [6], where two colors were generated considering the two different transition energies of the $\mathrm{Eu}^{2+}(\sim 2 \mathrm{eV})$ and $\mathrm{Eu}^{3+}(2.5 \mathrm{eV}-3 \mathrm{eV})$, respectively. However, very large operating voltages $(>105 \mathrm{~V})$ are needed to excite either $\mathrm{Eu}^{2+}$ or $\mathrm{Eu}^{3+}$, whereas a similar effect is obtained here with voltages five times lower (reducible by decreasing insulator thickness) and without rare-earth ion doping.

Finally, Fig. 4 depicts the integrated EL intensity versus voltage in accumulation and inversion regimes. Observe that the EL intensity increases with the applied voltage for both gate polarities. However, for accumulation it is larger than for inversion at $\pm 25 \mathrm{~V}$, which implies that the devices in accumulation are more effective in terms of optical power. This phenomenon suggests that by electrical pumping, $\mathrm{Si}$-ncs in $\mathrm{SiO}_{2}$ act as better luminescent centers than the $\mathrm{Si}$ nitride related defects (see Fig. 3).

In conclusion, a blue-green to near-IR switching EL has been demonstrated from an SRSO/SRSN bilayer structure by changing the gate dc voltage polarity in MNOSLED. The EL emission peaks in each spectrum were ascribed to Si-ncs into $\mathrm{SiO}_{2}$ and defects in $\mathrm{Si}_{3} \mathrm{~N}_{4}$. These results suggest that this structure allows the activation of two kinds of luminescent species due to the injection and transport properties of the whole bilayer. This work opens interesting perspectives on Si-based full-color microdisplays.

This work was supported by the Spanish Ministry of Science and Innovation (projects ICTS-NGG-31, TEC2009-08359, MAT2010-16407 and CSD2009-00013).

\section{References}

1. R. J. Walters, G. I. Bourianoff, and H. A. Atwater, Nat. Mater. 4, 143 (2005).

2. J. Warga, R. Li, S. N. Basu, and L. Dal Negro, Appl. Phys. Lett. 93, 151116 (2008).

3. M. Perálvarez, J. Barreto, J. Carreras, A. Morales, D. Navarro-Urrios, Y. Lebour, C. Domínguez, and B. Garrido, Nanotechnology 20, 405201 (2009).

4. Z. H. Cen, T. P. Chen, Z. Liu, Y. Liu, L. Ding, M. Yang, J. I. Wong, S. F. Yu, and W. P. Goh, Opt. Express 18, 20439 (2010).

5. A. Irrera, D. Pacifici, M. Miritello, G. Franzò, F. Priolo, F. Iacona, D. Sanfilippo, G. D. Stefano, and P. G. Fallica, Appl. Phys. Lett. 81, 1866 (2002).

6. S. Prucnal, J. M. Sun, W. Skorupa, and M. Helm, Appl. Phys. Lett. 90, 181121 (2007).

7. J. Barreto, M. Perálvarez, A. Morales, B. Garrido, J. Montserrat, and C. Domínguez, J. Mater. Res. 23, 1513 (2008).

8. J. M. Sun, W. Skorupa, T. Dekorsy, M. Helm, L. Rebohle, and T. Gebel, Appl. Phys. Lett. 85, 3387 (2004).

9. R. Huang, H. Dong, D. Wang, K. Chen, H. Ding, X. Wang, W. Li, J. Xu, and Z. Ma, Appl. Phys. Lett. 92, 181106 (2008).

10. S. M. Sze, Physics of Semiconductor Devices, 2nd ed. (Wiley, 1981).

11. Yu. V. Ryabchikov, P. A. Forsh, E. A. Lebedev, V. Yu. Timoshenko, P. K. Kashkarov, B. V. Kamenev, and L. Tsybeskov, Semiconductor 40, 1052 (2006).

12. M. V. Fischetti, D. J. DiMaria, S. D. Brorson, T. N. Theis, and J. R. Kirtley, Phys. Rev. B 31, 8124 (1985).

13. J. Robertson and M. J. Powell, Appl. Phys. Lett. 44, 415 (1984). 